\title{
New Management Technologies and Digital Business Transformation
}

\author{
Vladimir V. Godin*, Andrey A. Dashkov, Alla V. Blinnikova, Anna E. Terekhova, \\ Olga M. Danilina
}

Department of Information Technology, Institute of Information Technology, State University of Management, Moscow, Russia

* Corresponding author. Email: godin@guu.ru

\begin{abstract}
The purpose of this article is to review and analyze the main roles of information technology in the company: auxiliary, restructuring, and digital transformation. The role of information technologies in a company is determined by their degree of influence on the strategy and business model. The restructuring role of information technologies is characterized by the formation of the electronic economy, the consumer economy, the emergence of strategic alliances, value-added communities, and meta-markets, as well as other forms of economic and business organization with appropriate management.

The main hypothesis of the research is that information technologies of digital transformation provide the creation of an economy in the form of cyberphysical systems of interaction between the virtual and real parts of the world, which took the form of digital enterprises, then digital platform enterprises within the platform economy, then enterprises as digital ecosystems within the economy of digital ecosystems, and other similar phenomena. As a result of the transformative influence of information technologies on companies and the business environment, management tasks and technologies have changed. The article considers the evolution of changes in companies, business environment and management using traditional research methods such as historical retrospect, analysis, comparison and evaluation. Disruptive technologies such as blockchain, Big Data, knowledge management, Agile, Scrum, Teaming, and design thinking are considered and their impact on company management and the system of creating value for consumers is evaluated. A detailed diagram of the relationship between technologies, their essence and the benefits obtained from the use of these technologies is provided. The article concludes about the strategic nature of the ongoing changes and the dominant role of modern information technologies that have a global impact on all business processes occurring in companies on the path of digital transformation. A significant risk on the way to digital transformation is an acute lack of qualified personnel who can not only solve technical problems of technology implementation, but also imagine what impact they can have on the business as a whole, taking into account the social impact on the business environment. The perspective of the research relates to the fact that we are witnessing only the beginning of historical, large-scale and difficult to predict changes in literally all aspects of human life.
\end{abstract}

Keywords: information technologies, digital transformation, new management technologies, disruptive technologies, big data, Agile, Scrum, Teaming, design thinking

\section{INTRODUCTION}

The information nature of management is particularly evident in modern conditions - a drastic reduction in the duration of business processes, business virtualization, real-time decision-making, and much more. The nature of management and the role of information technology (it) and information systems (is) in companies of any form and sphere of business are changing.

In fact, now we can observe the transformative impact of it and IP on companies and their management in three forms: it and IP play a supporting role, restructuring, and the role of digital transformation technologies. In the first case, it and is, without changing the nature of the actions of company employees, strengthen them technologically, replacing part of their actions with actions of technologies and systems. In practice, this means transforming the daily 
activities of employees (their routine loops) through automation and Informatization. The management system, in this case, makes it possible to count faster, speed up reporting, and improve decision-making. There are no fundamental changes in the company's strategy or business model.

\section{METHODOLOGY}

The basis of the research was the classic works of prominent world scientists on both information technologies and value creation for consumers [1-5].

The article used traditional research methods such as the historical retrospective method, which allowed us to analyze the work and track the dynamics of management problems under the influence of information technologies $[1,4,6]$, analysis methods that allowed us to make a scheme that allows us to understand the relationship of technologies and their impact on the company's business processes, management in the "new" conditions and competitiveness [7-11].

\section{DISCUSSION}

Further development of it and the is formed on the basis of it creates a different role for them in terms of their impact on the business-restructuring. At this stage, companies ' strategies and business models are transformed. At first, this was manifested in the emergence of the electronic economy, with its business models (B2B, B2C, C2C, G2C, etc.), in the further formation of the concepts of the information economy [3, 4], the information economy [1, 2], the knowledge economy, etc. the management of companies faced new questions: how to evaluate the potential of e-business for a particular company; what factors and the nature of the problems determine the decision to enter or not enter eCommerce; when to do it; how to change the strategy, business model, organizational structure of the company as a result; in what order to make changes and how to maximize the benefits of e-Commerce.

Along with changes in business strategies, there has been a change in perceptions of the required business models. There was a request to form a business as a project implemented by a network of enterprises: a network enterprise [1], value-added communities [6], metamarkets [12]. And to manage the development of valueadded communities as an environment for effective innovation, with shared services and support processes, with the transition from improving individual companies to optimizing the network of companies. Another form of transformation of business models has become strategic alliances of companies, combining all the resources of these companies for the purpose of optimizing costs and results since IP. This is especially evident in the emergence of strategic alliances among passenger airlines.
At the same stage, there was a change in business priorities - after the era of production and the era of quality, business was forced to recognize the formation of the consumer era. For the company's management, this meant moving from mass production to flexible manufacturing, with products with unique properties in a mass production environment and ensuring that manufacturers were able to combine individual customer preferences with an efficient production and planning system.

The emergence of a number of technologies that are commonly called the basic technologies of digital transformation - the Internet, social networks and social resources, mobile devices, cloud computing, big data Analytics, the Internet of things, and then the formation and development of artificial intelligence and machine learning technologies, blockchain, digital twin, virtual and augmented reality, human-machine interaction, etc. provided a transformative role for it and formed the digital economy. In this economy," the so-called cyberphysical system acts as a production complex, a production system that creates products, services, provides life and convenience for people and the population, in which there is an integral interaction between the virtual and real parts of the world" [13]. Features of this economy: digital enterprises, often with completely deserted value chains; "smart" automation (artificial intelligence technologies, machine learning solutions and embedded solutions); flexible workforce (with the ability to solve special tasks, "fast learning", "ability to accelerate"); platform economy (platforms and ecosystems for organizations), connecting consumers to the development of new products at the earliest stage and accelerating feedback; and much more. New forms of implementation of the functions of States, organizations of industries and companies have emerged: smart power supply networks and grid energy; smart homes, districts, cities; joint consumption; productive economy; waste-free economy; integrated health care; and so on.

At the moment, technologies in the field of working with data (artificial intelligence, foggy computing, supercomputing technologies, identification technologies, modeling, blockchain technologies, neural networks, etc.), communicating robots, additive technologies and additive manufacturing and the Internet of things have formed the fourth industrial revolution. At the present time, we see the continued development of the technological foundations and dominant technologies of "Industry 4.0". First of all, these are new automation based on computerintegrated systems, cyber-physical systems, M2M (machine - to-machine) communication technologies of the industrial Internet of things, new design principles (oriented services, modularity, parametric design, personalized products, etc.), interoperability and decentralization, virtualization, real-time data accumulation and analysis, etc.

The development and application of all these technologies has a powerful transformative impact on business: 
reducing the time from an idea to its implementation in the market; competition for the speed of changing business models; ensuring flexibility and creating unique products in mass production; the transition to unmanned production and mass introduction of robotic technologies; the widespread use of cloud services; data analysis and evaluation based on Big Data technologies; the formation of "maker economy" based on 3D printers; the creation of Smart Factory; end-to-end automation and integration "from equipment to Ministry"; etc. All this together forms a new industrial platform in the economy.

The processes described above, on the one hand, form a new image of management - using data and making decisions in real time, applying the results of big data analysis in management, building a business on the basis of digital platforms and ecosystems, and on the other - put forward qualitatively different management tasks at all levels of economic management. We are talking about a new industrial structuring (new organizational forms, digital support for cooperation, digital integration of producers and consumers, etc.); formation of appropriate infrastructure (management of suppliers of key production technologies, suppliers of infrastructure solutions and services (telecommunications and cloud services, data accumulation and analysis, etc.), encouragement of industrial consumers); creation of a new type of digital production enterprises; formation of market mechanisms for encouraging innovation; solving problems of intellectual property management; and others.

Modern economic transformations need to assess the risks and consequences for society. It is obvious that the change of technological patterns leads to the formation of a different social structure of society and this requires special research [3, 4, 6]. Another significant task is the task of education-the formation of generations of "digital natives" of the new economy, combining managerial, engineering and other competencies with digital skills. Lack of qualified personnel is one of the significant risks. In addition, the new conditions require other concepts of human labor.

The changes in companies that are happening and should be happening are strategic, not tactical. You need to ensure a continuous process of change management, with a choice between a leadership strategy or a strategy of following the leader. These changes are manifested in different ways in companies of different sizes. In small and medium-sized businesses, the initiator of changes is the owner or Manager. In fact, the change agent is the project Manager. In a large business, you need to change the corporate culture, support the ability to change [2].

In fact, the company's digital leadership includes three components:

- strategy-digital business transformation;

- organization - management of the company digital business;

- innovation - strategic digital excellence.

Taking this into account, digital transformation is a company's transition to a digital enterprise by changing the strategy, business model and culture of the organization, implementing it and IP, expanding the boundaries and capabilities of the company and allowing it to form its own ecosystem (building its own ecosystem or fitting into existing ones). Management tasks ensuring the digital transformation of the company (defining and realizing the value of digital business transformation, forming a new business model, ensuring digital leadership in the company, training employees and searching for talent, transforming the it infrastructure for new tasks).

Conceptually, building a company's strategy can be described as the evolution of strategic ideas to a specific focus of the company in the following sequence: on resources; on the market position, on key competencies, on the business model; and finally, on the business ecosystem. The current state of this process is the company as an ecosystem. The evolution of technological platforms and business ecosystems can be represented in the following sequence of changes: e-mail, web site, ecommerce, e-business, networking, digital ecosystems.

\section{RESULTS}

The emergence and development of end-to-end technologies and accelerating globalization are radically changing approaches to value creation and destroying seemingly immutable economic laws. The world is undergoing a transition from an economy where demand creates supply to an economy where supply creates demand. The one who can make the best offer with the highest consumer value, the one who gets the win in the face of loyal consumers. Technologies allow you to personalize the offer as much as possible, considering the characteristics of individual consumption. Change is necessary for survival and growth. In parallel with the process of transformation of companies under the influence of it and IP, management tasks changed. Currently, we are seeing an increasing penetration and intertwining of digital and management technologies. They enrich and complement each other, creating not only a "new" economy, but also a "new" management.

The question is, have new forms and technologies of management appeared to solve the new problems? Are there disruptive technologies for management?

A few technologies play a disruptive role for management and the emerging properties of management. Next, we will consider the impact of these technologies on the properties of management.

Blockchain is a disruptive management technology.

Modern companies can be considered as systems built on information interaction with the environment - other companies, trade unions, the state, and on the information interaction of elements of the company itself (divisions). This means that companies during their operation form networks for the exchange of information and assets (tangible or intangible resources). Companies record this 
interaction using various documents (such as contracts) that are stored by the parties to the interaction and by intermediaries [14]. The possibility of fraud, opacity, corruption, errors, high costs and risks, and much more determine the vulnerability and inefficiency of such a system. A decentralized distributed database with a cryptographic key, built on blockchain technology, solves all these problems, because it allows you to get rid of intermediaries in agreements between companies and people.

The media hype around blockchain technology is currently focusing on its financial applications, primarily in the field of cryptocurrencies (cryptocurrencies themselves and ICOS). But this is only one of the decentralized applications of the blockchain. Another decentralized application is DAOs-decentralized autonomous organizations-decentralized Autonomous organizations (a platform on the blockchain for business deployment in the form of a closed decentralized solution for creating virtual enterprises with their own financial services).

Along with decentralized applications, private blockchain systems (various blockchain-based systems developed and supported by organizations to solve their tasks) and blockchain services (public services built on the blockchain) are being developed.

Now, it is possible to fix the construction of elements of the blockchain economy. At the state level, this means reducing the importance of paper documents confirming the facts of committing any actions, creating various registers, increasing transparency and reducing the possibility of fraud, reducing the need for regulatory authorities, auditors, and so on.

In business, Blockchain is a platform for effective models of organizational transformation. In the financial sphere, the use of blockchain is a response to the complexity and increase in the scale of operations, high load and an increase in the number of failures. Blockchain allows you to get rid of centralization and intermediaries, reduce costs, and speed up processing. In other areas of businessdigital identification, digital assets, smart contracts, a huge number of other applications. Surveys of companies show that the main benefits of the technology are expected to reduce operating costs (73\% of respondents), reducing the time of calculations $(69 \%)$, reducing risks $(57 \%)$, increase the possibility of obtaining additional income (51\%) [15]. Undoubtedly, the use of blockchain technology leads to the transformation of business strategies, business models and operations of companies [16]. It forms a different management [17]. At the same time, the emergence of blockchain technology was accompanied by the expectation of creating a new fair society and transparent business conditions without intermediaries, without risk, without fraud. This technology called for the choice of "public versus corporate", "self-regulating community "versus " centralization". However, blockchain is only a technology that should be needed by society and business. Its applicability is based on the network property of organizations using this technology, the need for a mechanism for achieving consensus among network participants to confirm transactions, the ability to scale prototypes, the correct formulation of application goals, and so on.

Management and Big Data.

One of the most powerful impact on the management of companies was the group of Big Data technologies. Big Data refers to a set of approaches, tools and methods for processing structured and unstructured data, characterized by a large volume and significant diversity, to obtain human-perceived results that are effective in the conditions of continuous growth of this data [18].

The emergence of an increasing number of data sources, the ability to store and process virtually unlimited amounts of data of any structure, as well as the development of data analysis and machine learning methods, have predetermined the emergence of Big Data technologies, and in the future, Data-driven companies. What has happened in the field of management in these data-oriented companies?

First, the transformation strategy: how to start working with big data, what specific problem in the company is related to big data, and how to solve it.

Secondly, the transformation of the company's business practices: Analytics is the main tool for managing the company; restructuring business processes for Big Data; regulating procedures for collecting, transmitting, storing and integrating data; investing in data accumulation, extracting information from accumulated data, creating special tools for Analytics; implementing Analytics aimed at the future: "Why?", "Who?" and "what next?"; implementing predictive scenarios and models; changing the decision-making process.

A significant step in the company's transformation is the selection of staff with analytical thinking, training in information analysis skills, and the formation of a special corporate culture (there is no authority authority, decision - making based on data analysis, cultivating research thinking, data is the task of all employees).

Third, the implementation of changes in the organizational structure - the creation of divisions dealing with Big Data. A non-trivial task of managing a Data-driven company is to account for and assess risks in the field of ethical problems of applying the results of big data analysis technologies.

Knowledge management and management.

The transformation of the economy of goods into the economy of knowledge, when knowledge for most industries acquires the role of a key competence and is a factor in the innovative development of organizations, has been realized. Knowledge in such an economic system acts as a resource, commodity, and capital. There are three driving forces in the knowledge economy: knowledge itself (intellectual capital) as a strategic factor; rapid and continuous changes in everything, creating uncertainty for companies; globalization of all economic activities, 
leading to global competition and business interdependence.

The knowledge economy required a revision of the principles of building economic and managerial relations in organizations, which resulted in the formation of a new type of management - knowledge management. The initial positions of knowledge management are ideas that emphasize the special role of man, his intelligence and knowledge. In such management, it is recognized that the values of the organization are not only its assets, goods/services produced, but also the competence of employees, the degree of commitment of consumers, culture, know-how and other components of the concept of "intellectual capital". Note that in knowledge management, priority is given to knowledge, considered, on the one hand, as the most important type of resource, and on the other - as the most valuable product of a modern organization. Therefore, the management problem more and more implies the management of intellectual capital and information.

An important condition for the transition to knowledge management is the infrastructure that companies need to create, consisting of knowledge bases and data and knowledge repositories; data and text mining tools; document, content and email management tools; external information flow management tools; teamwork tools; integrated knowledge management tools - knowledge management platforms and corporate knowledge portals; decision support tools-systems that support discussion groups, expert systems, etc.

Agile, Scrum and other innovations as new management technologies [16, 19].

As a response to the transformation of companies and the business environment, a number of innovations have emerged and are being successfully applied, which can be attributed to new management technologies: Agile, Scrum, Teaming, and design thinking.

In February 2001， 17 programmers released an Agile Manifesto containing the basic principles of agile software development:

- people and interaction are more important than processes and tools;

-a working product is more important than comprehensive documentation;

- cooperation with the customer is more important than agreement on the terms of the contract;

- readiness for change is more important than following the original plan.

The principles laid down in the Agile approach have formed a new view of management, in which the needs of the customer and ensuring its competitive advantage are paramount; encouraging changes; constant collaboration with the customer of motivated professionals in the right conditions; a working product is the main indicator of progress; constant attention to technical excellence and design quality; minimizing unnecessary work; selforganizing teams.
Scrum is a flexible method for developing a product in a complex, volatile, and uncertain environment. Main ideas of the method:

-you need a person who has the most complete understanding of the product;

- the project team should be Autonomous and include all the necessary specialists;

-team activities should be divided into short segmentssprints - with clear goals;

- the team's activities must be constantly improved.

Teaming is an approach to organizing the activities of large groups of people, based on the principle of psychological security and constant training to achieve increasingly complex and ambitious goals. It ensures coordination and collaboration without creating stable and inflexible organizational structures. This innovation in management has emerged in a situation where teamwork skills are even more relevant than before to survive in the era of digital transformation.

It has become necessary to move away from hierarchical systems and Directive management style to open flat structures that allow, first, to reduce the gap between the adoption and implementation of innovations (this is the goal of the concept of design thinking); second, to involve all team members in the development of the company; third, to eliminate the fear of mistakes and establish open competition between different points of view; and fourth, to coordinate actions for making common decisions [10]. The transition from "work teams" to "teaming" due to rapid change under the influence of technology, increasing uncertainty and complexity, speed decision task as a result of outsourcing, the instability of teams, and with constant experimentation and research. Often the solution of such tasks is not within the power of one leader and there is a need for shared leadership. When a Manager gives orders rather than controls, when he encourages flexibility rather than requiring compliance, then the organization goes to a new level, thus laying a solid Foundation for more effective work and the success of the company. Teams 'slogan in the era of digital transformation " Change people... Or change people."

Design thinking and the new look of the Manager.

Digital transformation can go in three directions:

- changing the customer experience by better understanding the unique and individual experience of each customer,

- automation/digitization/re-engineering of internal business processes,

- changing the business model.

For the successful implementation of any of the directions, you can not do without design thinking - "a new approach to the design of innovative solutions, focused on people, based on the tools used by designers and used to integrate the needs of people, technological capabilities and requirements for business success" [8]. In fact, this is a new round of development of the concept of consumer orientation to create value together with the consumer laid down by K. K. Prahalad, M. S. Krishnan V. 
Ramaswami [20, 21]. Design thinking becomes popular when the speed of change brought by end-to-end technologies has become so great that there is almost no time left for full-scale marketing research [11]. Design thinking methods allow you to quickly understand the current situation, generate ideas for new products or modify existing ones, conduct rapid prototyping and testing of proposed solutions with minimal costs.

So, if we want to follow existing patterns, then design thinking is not necessary. Design thinking is necessary to quickly create a unique product; that is, the opposite of benchmarking, i.e., the method of comparison with the best samples.

The first stage of design thinking is empathy. It is important to understand the real behavior of people, the reasons why they behave this way and not otherwise, their motivation, the picture of the world and as a result, their values and needs. It is empathy that becomes the key to opening opportunities, as it reveals consumer problems that require solutions. The next steps, if the consumer has been correctly understood, will lead to the promotion of creative ideas and finding possible solutions to be prototyped and tested.

Design thinking is becoming a mandatory tool for a modern Manager when conducting digital transformation as a way to quickly test hypotheses and find solutions to consumer problems.

\section{CONCLUSION}

Future: consequences of new management technologies. At the present stage, information technologies have provided the peak of revolutionary changes in business and society. The new technological structure of the economy, based on cyberphysical systems in which people and robots will work simultaneously, will lead to getting rid of routine and cheaper service operations, and optimizing business processes. Smart components will be added to most modern transaction systems (ERP Enterprise Resource Planning), BPMS - Business Process Management Systems, and EPM - Enterprise Project Management). A significant number of companies will use a full set of big data analysis and business Analytics tools in their decision-making process for any task, and above all, to improve the effectiveness of the process of creating marketing offers and improving the customer experience. These technological opportunities at the company level will require changes in management technologies, and at the society level will lead to the formation of a new social order and a different system of basic values.

We can anticipate how companies will change and how management will transform. And questions about how the structure of companies will change, how their work methods will change, how the roles of managers at different levels of the hierarchy will change, and whether hierarchical structures will still be in demand, have only tentative answers. But in a very short time, we will get answers to these questions and time will show how correct decisions were made today.

\section{REFERENCES}

[1] M. Castells, The Internet Galaxy. Reflections on the Internet, Business and Society, Oxford University Press, 2001.

[2] I.N. Chuev, T.M. Panchenko, V.S. Novikov, O.A. Konnova, N.G. Iraeva, I.S. Karabulatova, Innovation and Integrated Structures of the Innovations in Modern Russia, International Review of management and marketing 6(1S) (2016) 239-244.

[3] B. Gates, Business @ The speed of thought. Using a digital Nervous System, New York, 1999.

[4] I. Karabulatova, Kh. Vildanov, A. Zinchenko, E. Vasilishina, A. Vassilenko, Problems of transformation matrices modern multicultural identity of the person in the variability of the discourse of identity Electronic Information Society, Pertanika Journal of Social Science \& Humanities 25(S) (2017) 1-16.

[5] J.A. Slywotzky, J.D. Morrison, B. Andelman, The profit zone: how strategic business will lead you to tomorrow's profits, 2002.

[6] I. Karabulatova, O. Barabash, V. Barabash, N. Vinogradova, S. Kulikov, F. Izbassarova, Homo Ludens in modern postmodern discourse: new possibilities of manipulation of public consciousness, in: International Conference on Man-Power-Law-Governance: Interdisciplinary Approaches, Atlantic Press, 2019, pp. 48-50.

[7] R. Adner, J.E. Oxley, B.S. Silverman, Collaboration and Competition in Business Ecosystems, Emerald, 2013.

[8] T. Brown, Change by Design: How Design Thinking Transforms Organizations and Inspires Innovation Hardcover, September 29, 2009.

[9] E. Derby, D. Larsen, Agile Retrospectives: Making Good Teams Great, Pragmatic Bookshelf, 2006.

[10] C.A. Edmondson, Teaming: How Organizations Learn, Innovate, and Compete in the Knowledge Economy, Harvard Business School, Jossey-Bass, 2013. 
[11] J. Sutherland, Scrum: The Art of Doing Twice the Work in Half the Time, Crown Publishing Group, 2014.

[12] G. Means, D. Schneider, MetaCapitalizm The eBusiness Revolution and the Design of 21st-Century Companies and Markets, John Wiley \& Songs, 2000.

[13] J. Rifkin, The Third Industrial Revolution: How Lateral Power Is Transforming Energy, the Economy, and the World, St. Martin's Press, 2011.

[14] O. Gassmann, C. Michaela, K. Frankenberger, The Business Model Navigator: 55 Models That Will Revolutionise Your Business, 2014.

[15] K. Ordov, (2017), The gods of the blockchain technology could destroy the bureaucracy of the States, Available at: https://www.forbes.ru/tehnologii/344141bogi-blokcheyna-tehnologiya-mozhet-razrushitbyurokratiyu-gosudarstv.

[16] I.S. Karabulatova, M.S. Vykhrystyuk, N.G. Dolzhenko, E.I. Mychko, E.V. Potmenskaya, The Use of Stable Expressions in Modern Economic Discourse as an Emotional Increase in the Potential Impact on Purchasing Power, Space and culture, India 7(4) (2020) 283-292. DOI: https://doi.org/10.20896/saci.v7i4.488

[17] J.G. Mark, W. Wei, Business Ecosystems in China, 2017.

[18] Gartner Top 10 Strategic Technology Trends for 2018, Available at:

https:/www.gartner.com/smarterwithgartner/gartnertop-10-strategic-technology-trends-for-2018/.

[19] K. Rong, Y. Shi, Business Ecosystems:

Constructs, Configurations, and the Nurturing Process, 2014.

[20] C.K. Prahalad, M.S. Krishnan, The new age of innovation. Driving Cocreated Value Through Global Networks McGraw-Hill, 2008.

[21] C.K. Prahalad, V. Ramaswamy, The Future of Competition: Co-creating Unique Value with

Customers, Harvard Business School Publishing, 2004. 